\title{
Electronic states of trans-polyacetylene, poly(p-phenylene vinylene) and $s p$ - \\ hybridised carbon species in amorphous hydrogenated carbon probed by resonant Raman scattering
}

\author{
M. Rybachuk ${ }^{1,2 *}$ and J.M. Bell ${ }^{2}$ \\ ${ }^{1}$ Federal Institute for Materials Research and Testing (BAM), Division VI.4 Surface \\ Technology, Unter den Eichen 87, 12205 Berlin, Germany \\ ${ }^{2}$ Faculty of Built Environment and Engineering, Queensland University of \\ Technology, 2 George St, Brisbane, Qld 4001, Australia
}

\begin{abstract}
Inclusions of $s p$-hybridised, trans-polyacetylene $\left[\right.$ trans $\left.-(\mathrm{CH})_{\mathrm{x}}\right]$ and $\operatorname{poly}(p-$ phenylene vinylene) (PPV) chains are revealed using resonant Raman scattering (RRS) investigation of amorphous hydrogenated carbon $(a-\mathrm{C}: \mathrm{H})$ films in the near IR UV range. The RRS spectra of trans- $(\mathrm{CH})_{\mathrm{x}}$ core $A_{g}$ modes and the PPV CC-H phenylene mode are found to transform and disperse as the laser excitation energy $\hbar \omega_{L}$ is increased from near IR through visible to UV, whereas $s p$-bonded inclusions only become evident in UV. This is attributed to $\hbar \omega_{L}$ probing of trans- $(\mathrm{CH})_{\mathrm{x}}$ chain inhomogeneity and the distribution of chains with varying conjugation length; for PPV to the resonant probing of phelynene ring disorder; and for $s p$ segments, to $\hbar \omega_{L}$
\end{abstract}

\footnotetext{
* Corresponding author. Fax:+61 73138 4135. E-mail address: m.rybachuk@qut.edu.au (M. Rybachuk)
} 
probing of a local band gap of end-terminated polyynes. The IR spectra analysis confirmed the presence of $s p$, trans $-(\mathrm{CH})_{\mathrm{x}}$ and PPV inclusions. The obtained RRS results for $a-\mathrm{C}: \mathrm{H}$ denote differentiation between the core $A_{g} \operatorname{trans}-(\mathrm{CH})_{\mathrm{x}}$ modes and the PPV phenylene mode. Furthermore, it was found that at various laser excitation energies the changes in Raman spectra features for trans $-(\mathrm{CH})_{\mathrm{X}}$ segments included in an amorphous carbon matrix are the same as in bulk trans-polyacetylene. The latter finding can be used to facilitate identification of trans $-(\mathrm{CH})_{\mathrm{x}}$ in the spectra of complex carbonaceous materials. 


\section{Introduction}

Amorphous carbon (a-C) and diamond-like carbon (DLC) solids are characterised by a large variety of types and properties that stem from combinations of principally two hybridised forms of carbon $s p^{2}$ and $s p^{3}$ and, for carbon materials formed in presence of hydrogen, as for $a-\mathrm{C}: \mathrm{H}$, the resultant properties are also controlled by the hydrogen content. Isotropic materials like DLC or $a$-C can, in principal, contain inclusions of a basic polymer, the trans isomer of polyacetylene [trans $\left.-(\mathrm{CH})_{\mathrm{x}}\right]$ according to simulations by Bernasconi et al. [1]. This introduced the idea that $C-C$ bonds in bulk trans $-(\mathrm{CH})_{\mathrm{x}}$ undergo a gradual saturation via chain interlinking at high pressure, transforming into an $a-\mathrm{C}: \mathrm{H}$ solid, and on the other hand, earlier experiments by Arbuckle et al. [2] showed that $s p^{3}$ clustering occurs if defect concentrations in trans- $(\mathrm{CH})_{\mathrm{x}}$ reach sufficiently high level. These findings can be related to the energetic mechanism of $s p^{2}$ and $s p^{3}$ bonding formation in a hydrogenated DLC [3]. The presence of trans $-(\mathrm{CH})_{\mathrm{x}}$ in a carbonaceous solid was reported by López-Ríos et al. [4] for CVD synthesised diamond, and Dischler et al. [5] and Piazza et al. [6] identified trans- $(\mathrm{CH})_{\mathrm{x}}$ inclusions in low temperature synthesised $a-\mathrm{C}: \mathrm{H}$. Assignment of a Raman peak at $c a .1140 \mathrm{~cm}^{-1}$ to $\omega_{1} C-C$ in plane bending mode and a peak at $c a .1490 \mathrm{~cm}^{-1}$ to $\omega_{3} C=C$ stretching mode to those of trans $-(\mathrm{CH})_{\mathrm{x}}$ was, at first, uncertain, since solution synthesised trans $-(\mathrm{CH})_{\mathrm{x}}$ is known to be unstable at the elevated temperatures used in ordinary DLC deposition $[4,7,8]$. Some authors inferred that very short (less than $20 C=C$ units) temperature stable trans $-(\mathrm{CH})_{\mathrm{x}}$ segments are formed between the diamond grains during deposition $[4,9$, 10]. Isotopic substitution experiments by Kuzmany et al. [11] and Michaelson et al. [12] confirmed the assignment of the $\omega_{1}$ and $\omega_{3}$ modes to trans $-(\mathrm{CH})_{\mathrm{x}}$. Recently Teii 
et al. [13] made an effort to correlate the interaction between the hydrogen-rich plasma and the amount of trans $-(\mathrm{CH})_{\mathrm{x}}$ in nanocrystalline diamond thin-films. Conclusive results, however, were not obtained since at present, the means to quantitatively identify the amount and/or the ordering of trans $-(\mathrm{CH})_{\mathrm{x}}$ inclusions in a given $a$-C or DLC solid are not sufficiently defined.

The purpose of this work is to present the resonant Raman scattering (RRS) investigation of basic $a-\mathrm{C}: \mathrm{H}$ films in the near-infrared (NIR) to ultraviolet (UV) range, and to demonstrate that these films host trans- $(\mathrm{CH})_{\mathrm{x}}$ inclusions (chains) characterised by intrinsic ordering and variable conjugation length; and to show that films also contain $s p$-hybridised carbon species and inclusions of poly( $p$-phenylene vinylene) (PPV) [14]. The $s p$-bonded species considered are short hydrogenterminated polyyne chains. Here we obtain experimental and theoretical results that demonstrate differentiation between the Raman modes of trans $-(\mathrm{CH})_{\mathrm{x}}\left(\right.$ core $A_{g}$ modes) and the PPV phenylene mode in $a-\mathrm{C}: \mathrm{H}$. We illustrate that at various laser excitation energies $\left(\hbar \omega_{L}\right)$, the changes in Raman spectra features for trans- $(\mathrm{CH})_{\mathrm{x}}$ segments included in an amorphous carbon matrix of an $a-\mathrm{C}: \mathrm{H}$ are the same as in bulk transpolyacetylene. Figure 1 shows an example of the RRS for bulk trans-polyacetylene for different excitation laser wavelengths $\left(\omega_{L}\right)$. This figure shows as the $\hbar \omega_{L}$ is increased from deep-red to blue excitation, the RRS bands change gradually from narrow, slightly asymmetric lines into more complex two-peak bands, each consisting of an un-shifted primary peak and an upward shifted satellite portion which becomes the prominent feature of the band at blue excitation [15]. In $a-\mathrm{C}: \mathrm{H}$ too, the $\omega_{1}$ and $\omega_{3}$ trans $-(\mathrm{CH})_{\mathrm{x}}$ modes become transformed and change positions, (disperse [9]), shapes and intensities with changing $\hbar \omega_{L}$, and these transformations strongly depend on the inherent degree of inhomogeneity of trans- $(\mathrm{CH})_{\mathrm{x}}$ chains. 


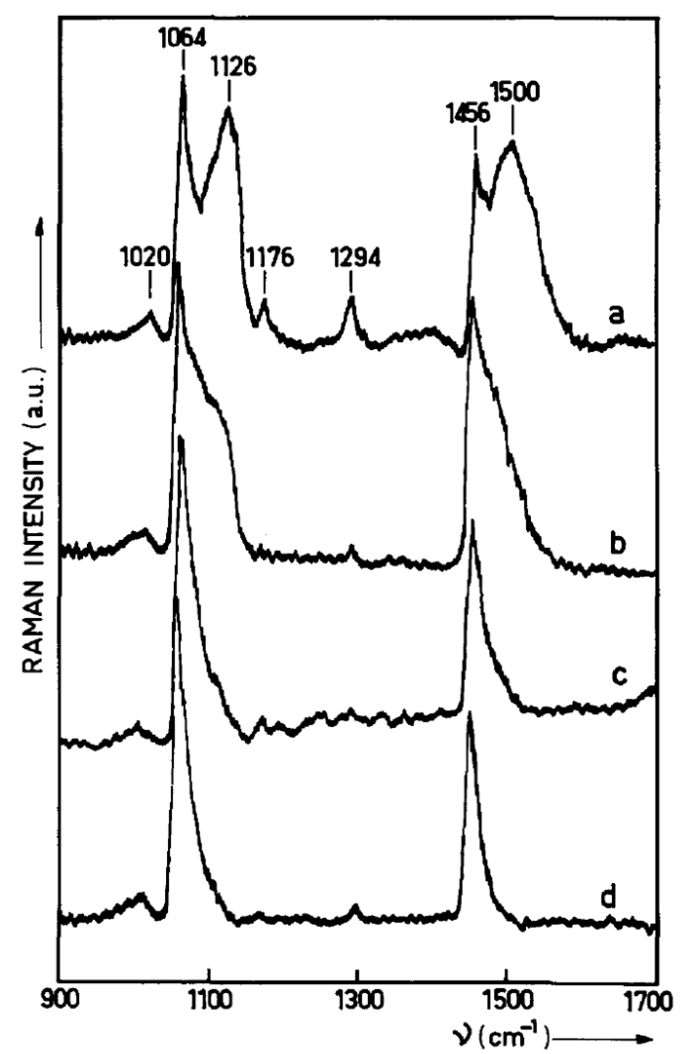

Fig. 1 - Resonant Raman spectra of bulk trans- $(\mathrm{CH})_{\mathrm{x}}$ at $78 \mathrm{~K}$ taken for different laser excitation wavelengths. a) $\omega_{L}=457.9 \mathrm{~nm}$; b) $\omega_{L}=514.5 \mathrm{~nm}$; c) $\omega_{L}=600 \mathrm{~nm}$; d) $\omega_{L}$ $=676.4 \mathrm{~nm}$, adapted from Ref. 15 .

The distribution of trans- $(\mathrm{CH})_{\mathrm{x}}$ segments with varying degrees of inhomogeneity (conjugation chain length, bond disorder) was computed employing the bi-modal chain distribution model proposed by Brivio and Mulazzi et al. $[8,15,16]$ and the amplitude mode theory proposed by Ehrenfreund et al. [7]. We aim to elucidate a simple approach that is needed to facilitate extraction of trans $-(\mathrm{CH})_{\mathrm{x}}$ contributions from core Raman spectra of $a-\mathrm{C}$, DLC or any other carbonaceous materials. 
The RRS has been successfully used to study inhomogeneity and disorder in amorphous carbon systems consisting of arbitrary combinations of $s p, s p^{2}$ and $s p^{3}$ hybridised states $[3,7,9,17-19]$. In this work the excitation energies over a wide range of energies from $1.58 \mathrm{eV}$ to $5.08 \mathrm{eV}$ were used, ensuring that the vibrational densities of states (VDOS) of the great majority of $s p^{3}, s p^{2}$ and $s p$ carbon mixtures are measured.

In an environment where energetic hydrogen ions are present, the probability for carbon atoms to enter into $s p$-type arrangement as either polyyne $(-C \equiv C-)_{n}$, a semiconductor, or polycumulene $(=C=C=)_{n}$, a semi-metal [20], is exceedingly low, since both species are highly unstable to hydrogen exposure [21] and temperature sensitive $[5,22,23]$. Identification of $s p$-hybridised inclusions in the hydrogenated $s p^{2}-s p^{3}$ aggregates are therefore, highly notable, since the $s p$ self-organisation mechanism, even at present, remains largely unresolved [24-28]. We focused on detection of $s p$-hybridised segments using the RRS process, and critically consider recent findings by D'Urso et al. [19]. In addition, infra-red (IR) absorption spectra analysis was used to identify $s p$ and trans $-(\mathrm{CH})_{\mathrm{x}}$ species.

\section{Experimental}

Deposition of $a-\mathrm{C}: \mathrm{H}$ films were performed on $\mathrm{Si}<111>$ wafers using a Helmholtz-type inductively coupled plasma (ICP) reactor operated on $\mathrm{CH}_{4} / \mathrm{Ar}$ mixture at temperatures of $380-400 \mathrm{~K}[29,30]$. The pressure was $6 \times 10^{-2} \mathrm{~Pa}$ and the substrate was DC negatively biased at -250 to $-300 \mathrm{~V}$. The use of substrate bias this range was found to have adverse effects to the formation of $s p$ or trans $-(\mathrm{CH})_{\mathrm{x}}$ 
segments. The formation of trans $-(\mathrm{CH})_{\mathrm{x}}$ and $s p$ bonded segments appears to be facilitated by the amount of atomic hydrogen in hydrocarbon plasma, and the ratio of $55 \% \mathrm{CH}_{4}$ to $45 \% \mathrm{Ar}$ was found to be most favourable in this work. Deposition was performed at extremely low rate of $\sim 30 \mathrm{~nm} /$ hour in a high density plasma, with the aim of obtaining high ordering of $s p^{2}$ phase, and allowing for a higher concentration of free radicals and a higher degree of gas phase reaction taking place.

The fabricated films were found to be of low intrinsic compressive stress $\leq 1$ GPa as determined using Stoney's equation [31] from the substrate curvature with hardness of approximately $20 \mathrm{GPa}$ and, a friction coefficient of 0.07 at $70 \%$ humidity measured by a nano-mechanical testing (UMIS). Electrical resistivity was in the range of $10^{8}-10^{9} \Omega \mathrm{cm}^{-1}$ as measured by using a four-probe testing method. Films were $\sim 140 \mathrm{~nm}$ thick with a maximum refractive index of 2.2 in the UV - blue region, as measured by IR - UV spectroscopic ellipsometry (J. A. Woollam Co.). The hydrogen content in the films was determined from the analysis of IR absorption spectra as used by Liu et al. [32] (normal mode vibrational frequency calculations) and from the analysis of UV Raman spectra $\left(\omega_{L}\right.$ of $\left.244 \mathrm{~nm}\right)$ as proposed by Casiraghi et al. [33] where the full width at half maximum (FWHM) of the $G$ peak, $G$ peak position and the dispersion of this peak at respective $\hbar \omega_{L}$ were used. This gave a hydrogen content of approximately $27( \pm 2.5)$ at $\%$.

The IR spectra in the range $3400-2600 \mathrm{~cm}^{-1}$ range were obtained using Nicolet Nexus Fourier transform infra-red (FT - IR) spectrometer operated in transmission mode with subtraction of $S i$ substrate background. For IR measurements, the same group (thickness, lattice orientation, and surface finish grade and backside surface roughness) of uncoated $\mathrm{Si}$ substrates was used, as for the film deposition experiments. 
Standard Gaussian peak functions were used to fit the constituent bands in the selected spectral range after linear background subtraction.

X-ray photoelectron spectroscopy (XPS), using Kratos AXIS Ultra photoelectron spectroscope with a monochromated Al $K_{\alpha} 1486.6 \mathrm{eV}$ X-ray source, was used ex situ to obtain $C_{1 \mathrm{~s}}$ spectra. The chamber vacuum level was maintained below $2.5 \times 10^{-9} \mathrm{~Pa}$ and the spectrometer was calibrated by peak referencing of $\mathrm{Au}$ $4 \mathrm{f}_{7 / 2}$ (binding energy $=84.0 \mathrm{eV}$ ) with respect to the Fermi level. XPS measurements were collected centred at $284.0 \mathrm{eV}$ at pass energy of $40 \mathrm{eV}$, with a resolution of 0.05 $\mathrm{eV}$ and dwell time of $250 \mathrm{msec}$; a total of 3 collection sweeps were used. Charge neutraliser was off and surface charging was not observed during the measurements. Information about the relative abundance of carbon hybridised fractions in the examined $a-\mathrm{C}: \mathrm{H}$ materials was obtained by decomposition of $C_{1 \mathrm{~s}}$ core electron binding energy spectra onto three constituent peaks: $s p, s p^{2}$ and $s p^{3}$. After the subtraction of Shirley background [34], Pearson VII line-functions corresponding to these peaks were fitted into the main $C_{1 \mathrm{~s}}$ peak employing the constrained fitting procedure, where an $s p$ constituent was fitted with an assigned binding energy (position) [35, 36] and, $s p^{2}$ and $s p^{3}$ constituents were fitted restricted to their respective energy separation gap [37] then, the value ratio of $s p / s p^{2} / s p^{3}$ was obtained based on integrated peak areas for the three hybridised line-functions. The presence of $s p$-hybridized species revealed by means of the XPS $C_{1 \mathrm{~s}}$ analysis was verified by analysing $244 \mathrm{~nm}$ Raman [19, 38, 39] and IR [5] results, and the $s p^{3}$ content by using 244 nm Raman results [17, 33].

Unpolarised Raman spectra across the excitation energy range $5.08 \mathrm{eV}$ to 1.28 $\mathrm{eV}$ were obtained ex situ at $293 \mathrm{~K}$ using 244, 532, 633, and $785 \mathrm{~nm}$ Renishaw instruments and 325 and $442 \mathrm{~nm}$ Kimmon Raman instruments. All excitation 
wavelengths excluding $785 \mathrm{~nm}$ were pulsed; the $785 \mathrm{~nm}$ was a continuous wavelength laser source. The frequency-doubled $\mathrm{Ar}$ ion laser was used for $244 \mathrm{~nm}, \mathrm{He} / \mathrm{Cd}$ for 325 and $442 \mathrm{~nm}$, the frequency-doubled YAG laser was used for $532 \mathrm{~nm}, \mathrm{He} / \mathrm{Ne}$ gas laser was used for $633 \mathrm{~nm}$, and a diode laser source was used for $785 \mathrm{~nm}$ excitations. All measurements were taken in dynamic mode with a specimen moved linearly at speeds of up to $30 \mathrm{~m} / \mathrm{s}$ and laser power was kept at or below $1 \mathrm{~mW}$ for all wavelengths minimizing the thermal damage. The acquisition time was varied between $10 \mathrm{~s}$ to 120 $\mathrm{s}$ and the spectral resolution was $1 \mathrm{~cm}^{-1}$.

There were two main options for fitting of the Raman spectra as noted by Casiraghi et al. [33]: an all Gaussians fit to Raman constituent bands, or a fit with a Breit-Wigner-Fano (BWF) line shape for the $G$ peak and a Lorentzian for the $D$ peak. The $a-\mathrm{C}: \mathrm{H}$ samples selected for this study did not display significant photoluminescence (PL) background, nonetheless, we find that the use of the BWF line is not the most appropriate since the BWF $Q$ coupling coefficient is influenced by the PL background, and the BWF lineshape tends to adjust its asymmetry reproducing a part of the PL slope $[33,40]$. This does not lead to reproducible fitting of the Raman spectra. Alternatively, fully symmetric Gaussian line-shapes provide better, reproducible fit in the presence of a PL background. In the Raman spectra presented, the linear PL background was subtracted and all constituent peaks were fitted with Gaussian line-shapes using a nonlinear least squares fitting procedure [41].

\section{Results and discussion}

\subsection{Identification of $\pi$ - conjugated polymeric inclusions in $a-C: H$}


Figure 2 shows the RRS spectra of an examined $a-\mathrm{C}: \mathrm{H}$ film with PL background subtracted and fitted with Gaussian line-shapes to the constituent peaks.

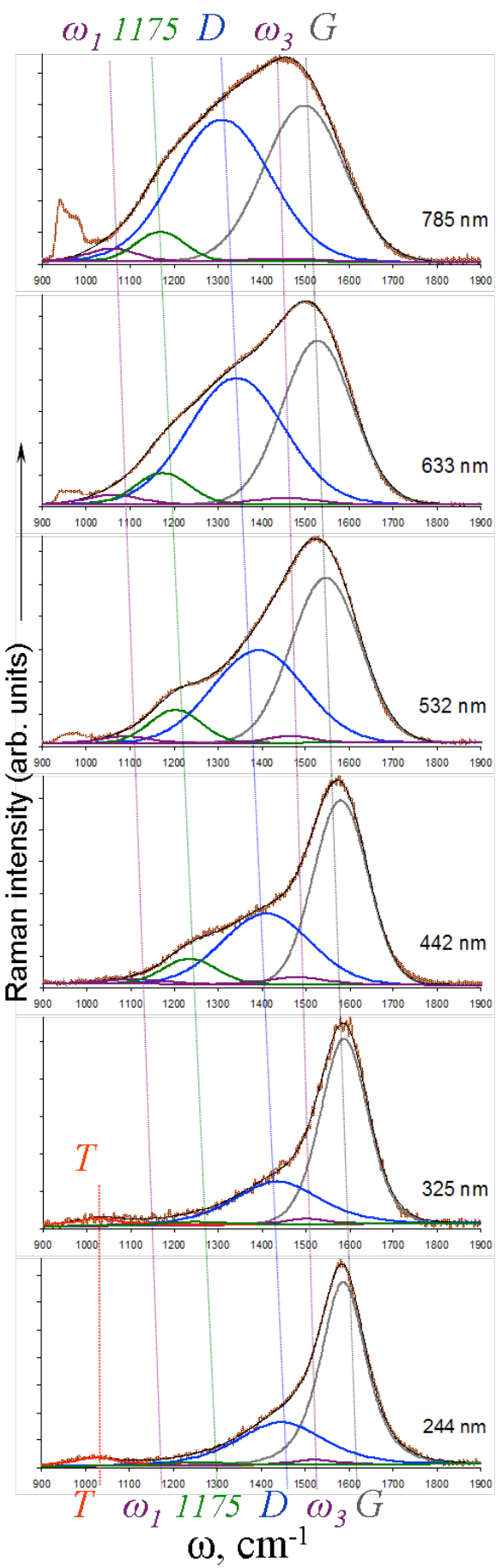


Fig. 2 - Resonant Raman spectra of $a-\mathrm{C}: \mathrm{H}$ at $293 \mathrm{~K}$ showing contributions from trans$(\mathrm{CH})_{\mathrm{x}}\left(\omega_{1}\right.$ and $\left.\omega_{3}\right)$ modes, PPV $\left(1175 \mathrm{~cm}^{-1}\right.$ mode $)$, and DLC ( $D, G$, and $T$ modes $)$. An asymmetric peak visible at NIR-visible (green) $\hbar \omega_{L}$ at $950 \mathrm{~cm}^{-1}$ is the second order Si, from Ref. 14.

The fitted bands are common DLC $D$ and $G$ modes for NIR and visible and the $T$ mode for UV $\hbar \omega_{L}$ excitations [17]; and the two $A_{g}$ zone center vibrational modes of trans $-(\mathrm{CH})_{\mathrm{x}}$, the $\omega_{1}$ and $\omega_{3}[7,18,42]$. The weak $\omega_{2}$ mode that usually appear at 1275 $-1295 \mathrm{~cm}^{-1}$ range (a peak labelled '1294', Fig. 1) was not present, nor the peak corresponding to the $B_{g}$ mode of trans $-(\mathrm{CH})_{\mathrm{x}}$ that is normally observed in $1000-$ $1100 \mathrm{~cm}^{-1}$ range (a peak labeled '1020', Fig. 1), however their contributions could be hidden by the tails of the fitted $D$ and the $\omega_{1}$ bands. Fundamentally, the absorption for bulk trans- $(\mathrm{CH})_{\mathrm{x}}$ occurs within $1.5-1.7 \mathrm{eV}$ range and corresponds to the zone centre $A_{g}$ Raman modes at opening frequencies of 1060, 1280 and $1450 \mathrm{~cm}^{-1}[18,42]$. That is at N-IR $\hbar \omega_{L}$. As the Raman excitation energy increases, and therefore moves away from the band gap resonance, the Raman sidebands exhibit radical lineshape changes as illustrated in Fig. $1[15,18,43,44]$. Shoulders appear at the high frequency side of the primary $\omega_{1}$ and $\omega_{3}$ modes that eventually extend into secondary peaks at excitation energies well above the band gap at $\hbar \omega_{L}=2.71 \mathrm{eV}$ [18]. The RRS spectra disperses [9] and the resultant trans- $(\mathrm{CH})_{\mathrm{x}}$ peaks change intensities, $I$ and widths $\Gamma$, and the overall spectrum is in addition affected by light polarisation $[45,46]$. The RRS of trans- $(\mathrm{CH})_{\mathrm{x}}$ secondary peaks, such as the peaks appearing at $\omega_{L}$ of $457.9 \mathrm{~nm}$ (noted as '1126' and '1500', Fig. 1) in bulk samples become more pronounced at higher excitation energy. However, the complexity of separating trans- $(\mathrm{CH})_{\mathrm{x}}$ from the 
host DLC modes leads us to analyse a single symmetric band distribution [43]. This approach was proved by Ehrenfreund et al. [7] to be sufficient to account for a double peak Raman structure.

Together with common DLC and trans- $(\mathrm{CH})_{\mathrm{x}}$ modes we find a peak positioned at $1175 \mathrm{~cm}^{-1}$ when probed by $785 \mathrm{~nm} \omega_{L}$ which we assign to a $C C-H$ bending mode of the ring in neutral poly ( $p$-phenylene vinylene) (PPV) [47, 48]. The origin of this mode could, in fact, be due to defects in $s p^{2}$ aromatic rings since in single crystals, only phonons with the wave vector rule $k=0$ contribute to Raman scattering. Defects lead to relaxation of this selection rule and therefore provide means for phonons from outside the centre of the Brillouin zone to contribute to the Raman scattering. If this $1175 \mathrm{~cm}^{-}$ ${ }^{1}$ mode indeed belongs to PPV chains, the other PPV zone centre vibrational modes found at higher frequencies in $1200-1330$ and $1540-1625 \mathrm{~cm}^{-1}$ range will certainly be obscured by the host $D$ and the $G$ modes [49]. Owning to its large width, $\Gamma_{1175}$ the $1175 \mathrm{~cm}^{-1}$ vibrational mode could be effectively a combination of vinylene and a $C C-$ $H$ ring bend modes since the zone mode frequency for vinylene is at approximately $1145 \mathrm{~cm}^{-1}$ [47]. As the Raman excitation energy increases from NIR to UV range all peak positions shift to a higher vibrational frequency obeying phonon confinement rules [17], as shown in Figure 3(a) where peak dispersion, $\Delta \omega$ is denoted as the shift in a peak position relative to base position at NIR excitation $\left(\hbar \omega_{L}=1.58 \mathrm{eV}\right)$. Figure 3(b) summarizes changes in widths for all fitted peaks. The gradual decrease in $I(D) / I(G)$, the intensity ratio for the $D$ and $G$ peaks, from $\sim 0.9$ to 0.2 , the pronounced reduction in $\Gamma_{D}$ and $\Gamma_{G}$, and the $G$ peak saturating [17] at approximately $1590 \mathrm{~cm}^{-1}$ for $244 \mathrm{~nm}$ excitation indicate that $a-\mathrm{C}: \mathrm{H}$ films hosting trans- $(\mathrm{CH})_{\mathrm{x}}$ and PPV inclusions consist of a highly ordered and symmetric $s p^{2}$ phase $[17,30]$. 


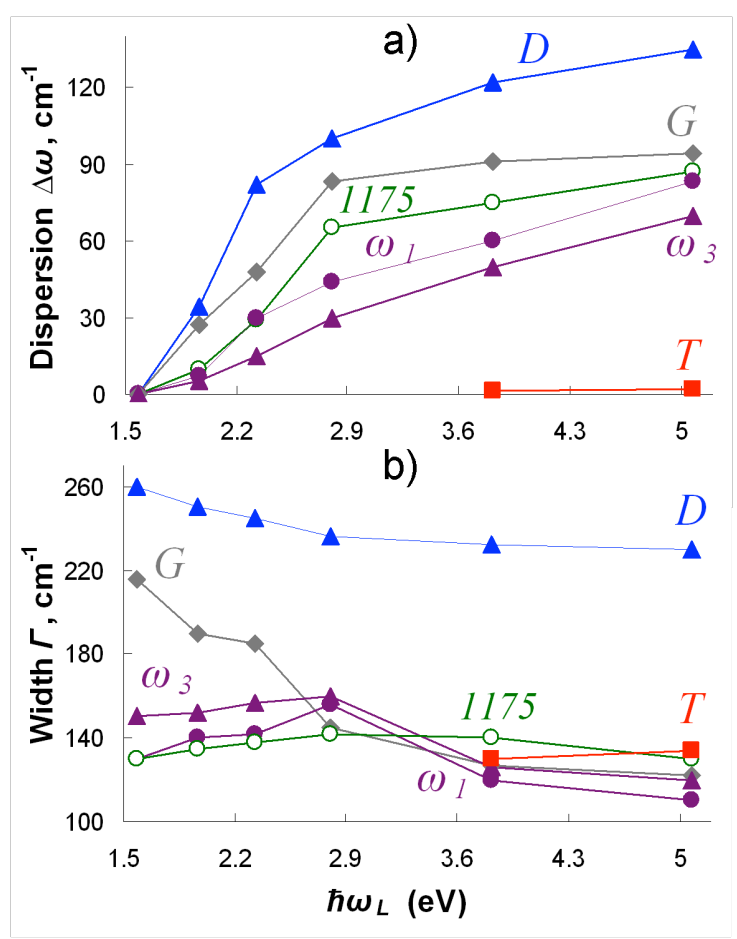

Fig. 3 a) - Peak dispersion, $\Delta \omega$ and b) - peak widths, $\Gamma$ for all constituent peaks as a function of the laser excitation energy $\hbar \omega_{L}$, from Ref. 14.

There is no $T$ peak dispersion at higher excitation energies in agreement with earlier reports [17]. The band gap for PPV is in the range of $2.2-2.5 \mathrm{eV}[48,50]$ and therefore it is selectively probed by excitation energy corresponding to green light $\left(\omega_{L}\right.$ of $532 \mathrm{~nm}$ ). Figure 4 illustrates relative changes of the fitted $\omega_{1}, \omega_{3}$ and $1175 \mathrm{~cm}^{-1}$ bands in the spectra of $a-\mathrm{C}: \mathrm{H}$ as a function of $\hbar \omega_{L}$. 


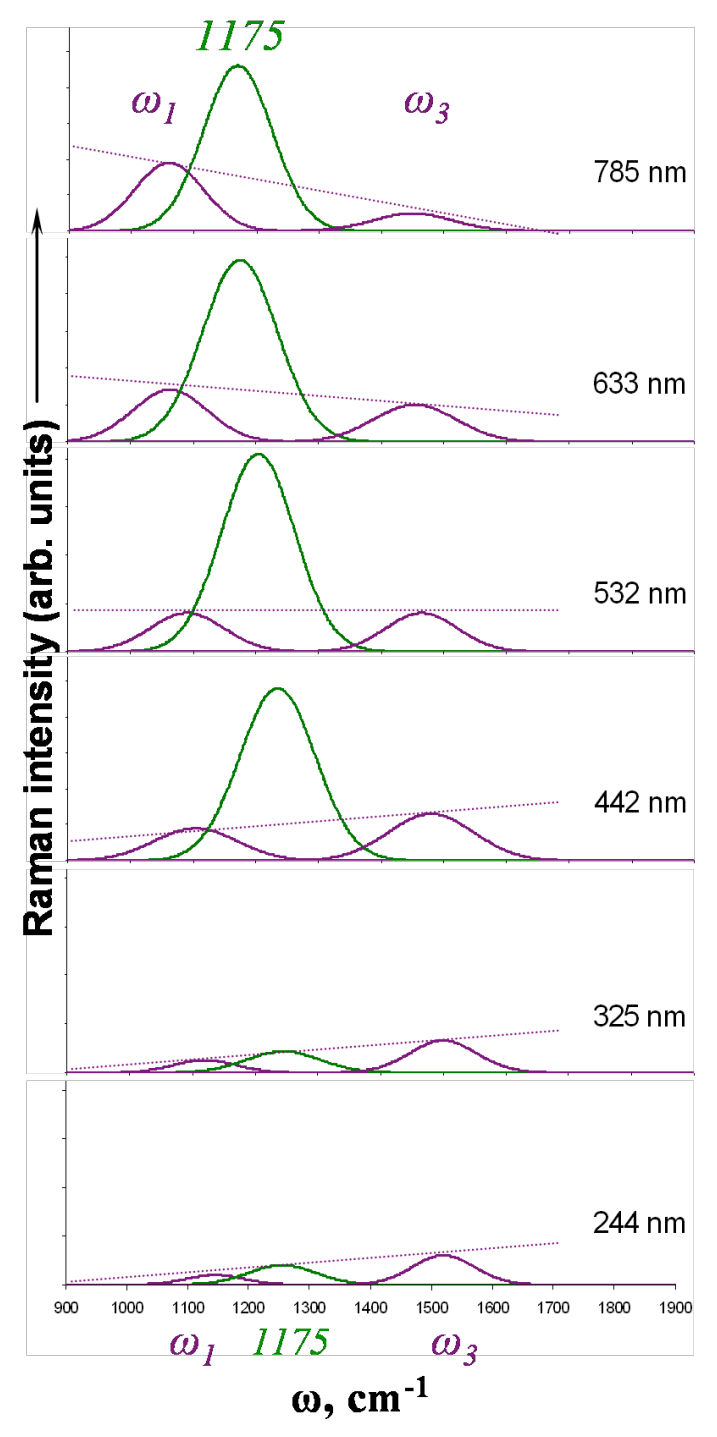

Fig. 4 - Changes in the fitted $\omega_{1}, \omega_{3}$ and $1175 \mathrm{~cm}^{-1}$ bands (intensity magnified by a factor of 5) relative to the laser excitation energy $\hbar \omega_{L}$ in the spectra of $a-\mathrm{C}: \mathrm{H}$. Light dotted line over $\omega_{1}$ and $\omega_{3}$ bands denotes the $I\left(\omega_{3}\right) / I\left(\omega_{1}\right)$ trend.

Figure 5(a) illustrates changes in the relative intensity of the $1175 \mathrm{~cm}^{-1}$ peak, $I(1175)$ at different excitation energies; $I(1175)$ is calculated as intensity of the 1175 peak over the total intensity of all constituent peaks including the intensity of the $T$ peak in the UV Raman spectra. 


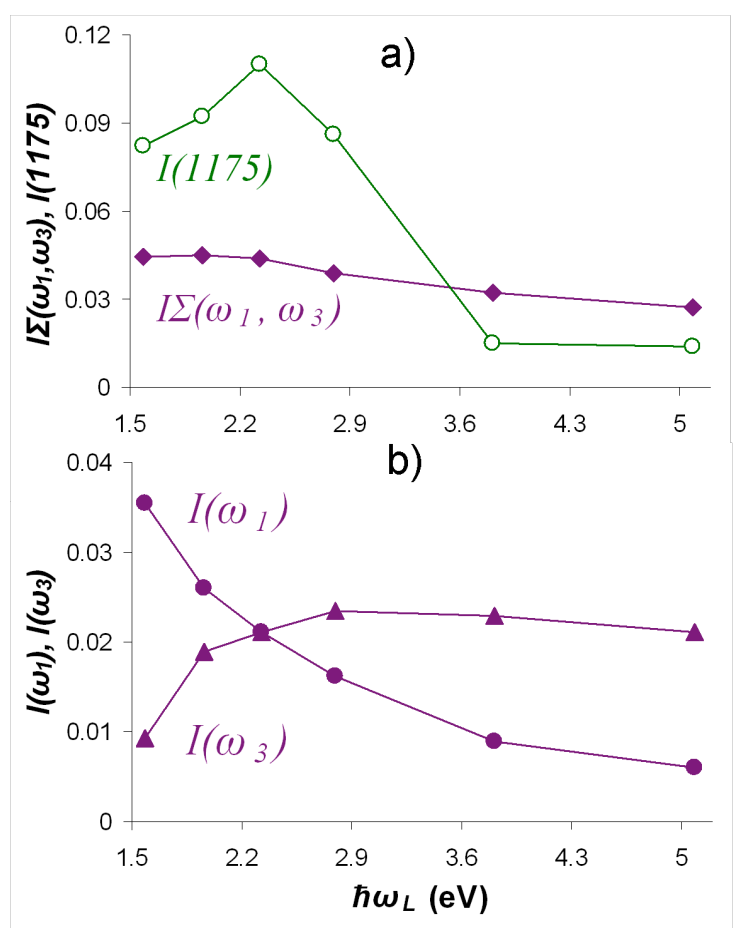

Fig. 5 a) - Evolution of relative intensities of the $1175 \mathrm{~cm}^{-1}$ peak, $I(1175)$ and trans$(\mathrm{CH})_{\mathrm{x}}$ contributions, $I \Sigma\left(\omega_{1}, \omega_{3}\right)$ and b) relative intensities of $\omega_{1}, I\left(\omega_{1}\right)$ and $\omega_{3}, I\left(\omega_{3}\right)$ peaks as a function of the laser excitation energy $\hbar \omega_{L}$.

The $I(1175)$ reaches its highest intensity position at $\hbar \omega_{L}=2.3 \mathrm{eV}$ as revealed in Fig. 4, although Fig. 3(b) shows that changes in the peak width, $\Gamma_{1175}$ are minor at this band bap frequency. This PPV peak at $1175 \mathrm{~cm}^{-1}$ is certainly of $s p^{2}$ origin since its contributions disappear in UV excitation.

The total relative intensity for trans- $(\mathrm{CH})_{\mathrm{x}}$ contributions calculated as sum of relative intensities of $\omega_{1}, I\left(\omega_{1}\right)$ and $\omega_{3}, I\left(\omega_{3}\right)$ peaks and denoted as $I \Sigma\left(\omega_{1}, \omega_{3}\right)$ is shown in Fig. 5(a). The magnitude of $I \Sigma\left(\omega_{1}, \omega_{3}\right)$ gradually decreases from N-IR to UV $\hbar \omega_{L}$. Individual trends of $I\left(\omega_{1}\right)$ and $I\left(\omega_{3}\right)$ contributions are shown in Fig. 4 and 
Fig. 5(b). The trans- $(\mathrm{CH})_{\mathrm{x}}$ features as $I\left(\omega_{1}\right)$ and $I\left(\omega_{3}\right)$ intensities (Fig. 4 and Fig. 5(b)) and peak widths, $\Gamma$ (Fig. 3(b)) for the $\omega_{1}$ and $\omega_{3}$ peaks exhibit strong transformations as the excitation energy increases from NIR excitation to blue excitation, but is most pronounced with excitation energy corresponding to green light; that is $I\left(\omega_{1}\right)$ and $I\left(\omega_{3}\right)$ intensities becoming inversely related with a fall in the total intensity, $I \Sigma\left(\omega_{1}, \omega_{3}\right)$ and $\Gamma_{\omega 1}$ and $\Gamma_{\omega 3}$ achieve a maximum in the blue-green excitation region. These are not related to tuning into the band gap frequency for trans $-(\mathrm{CH})_{\mathrm{x}}$ that requires much less energy (in the NIR $[18,42]$ ), but have been regarded as evidence of the presence of inhomogeneity (disorder) in trans- $(\mathrm{CH})_{\mathrm{x}}$ chains.

The disorder is due to a distribution of the electronic energy gaps and their respective frequencies; these are selectively probed via the variation of $\hbar \omega_{L}$ of RRS process, and result in the shift and broadening of phonon bands [7]. Major attempts to describe the inhomogeneity of trans- $(\mathrm{CH})_{\mathrm{x}}$ via a distribution of chains with varying length of $\pi$-electron conjugation [51] employed a particle-in-the-box [52] and Hückeltype calculations [53]. The bi-modal distribution model proposed by Brivio and Mulazzi et al. $[8,16]$ suggested a double peak distribution to arise from individual contributions of both long and short trans $-(\mathrm{CH})_{\mathrm{x}}$ segments that show unequal resonant enhancement at a given excitation energy. The inhomogeneity of trans- $(\mathrm{CH})_{\mathrm{x}}$ could also be described employing the distribution of the electron-phonon coupling constant $\lambda, p(\lambda)[7,54]$ of the amplitude mode (AM) theory proposed by Ehrenfreund et al. [7]. When other parameters are fixed, $\lambda$ determines the Peierls relation for the energy gap $E_{g}(\lambda)=W \exp (-1 /(2 \lambda))$ and laser frequencies, $\omega_{L}$, and where $W$ is the width of the $\pi$ band. The maximum for $p(\lambda)$ occurs at $\lambda=\lambda_{0}$, whereas resonance induced changes in peak position, $I$ and $\Gamma$ result from the condition $\hbar \omega_{L}=E_{g}(\lambda)>E_{g}\left(\lambda_{0}\right)$. We applied the AM model to study trans- $(\mathrm{CH})_{\mathrm{x}}$ inclusions in $a-\mathrm{C}: \mathrm{H}$ and the obtained results yielded $\lambda$ 
distribution range from $\sim 0.17$ for NIR to $\sim 0.24$ for UV, in good agreement with the AM model. The distribution of $\lambda$ rises from finite localisation lengths and bond length disorder, consequently AM theoretical calculations indicate that trans- $(\mathrm{CH})_{\mathrm{x}}$ segments probed by higher $\hbar \omega_{L}$ are of shorter $\pi$ - conjugation lengths and of higher bond disorder. The AM findings are complemented by calculations determining the length of the segments using Brivio and Mulazzi bi-modal distribution model which offers empirical relations for dependence of the conjugation length (long and short) on the frequency of the $\omega_{1}$ and $\omega_{3}$ modes. It was found that the approximate length for both single $C-C$ and double $C=C$ bonds in probed trans $-(\mathrm{CH})_{\mathrm{x}}$ segments is no less than 120 bond lengths units at the estimation limit of the model; and to a minimum of approximately 8 . Shorter chains are probed by higher excitation energies, as illustrated in Figure 6 [16] for the functional dependence of the $\pi$ - electron gap, $\Omega$, eV and the relative optical absorption on the chain lengths of trans $-(\mathrm{CH})_{\mathrm{x}}$ segments.

The average chain population is $\sim 25( \pm 5)$ bond length units owing to the uncertainties given by the Raman fitting and the bimodal distribution model [8]. All trans $-(\mathrm{CH})_{\mathrm{x}}$ chains included in $a-\mathrm{C}: \mathrm{H}$ are highly disordered as evidenced by wide $\omega_{1}$ and $\omega_{3}$ Raman peaks reaching their maximum in the blue-green range, shown in Fig. $3(b)$.

We have calculated the theoretical distribution for $I\left(\omega_{3}\right) / I\left(\omega_{1}\right)$ vs. $\hbar \omega_{L}$ independent of a given trans- $(\mathrm{CH})_{\mathrm{x}}$ chain length using the AM formalism that was previously completed by Ehrenfreund et al. [7] for the visible laser excitation to include N-IR and UV $\hbar \omega_{L}$. Fitting of $\omega_{1}$ and $\omega_{3}$ spectral constituents delivered the inverse relationship between the $I\left(\omega_{3}\right)$ and $I\left(\omega_{1}\right)$ parameters, $I\left(\omega_{3}\right) / I\left(\omega_{1}\right)$ relative to the laser excitation energy as illustrated in Fig. 4 and Fig. 5(b). Figure 7 shows that our experimental results obtained for the varying $\hbar \omega_{L}$ are in good agreement with the 
theoretical distribution predicted by the AM model and with Ehrenfreund's experimental data. The observation of relative intensities of $\omega_{1}$ and $\omega_{3}$ bands, $I\left(\omega_{1}\right)$ and $I\left(\omega_{3}\right)$ (Fig. 4), and the theoretical distribution of $I\left(\omega_{3}\right) / I\left(\omega_{1}\right)$ ratio (Fig. 7) relative to $\hbar \omega_{L}$ evidences that $\omega_{1}$ and $\omega_{3}$ peak resonance responses to excitation energy of green laser are essentially equal. These are indicated by equal magnitudes of $I\left(\omega_{1}\right)$ and

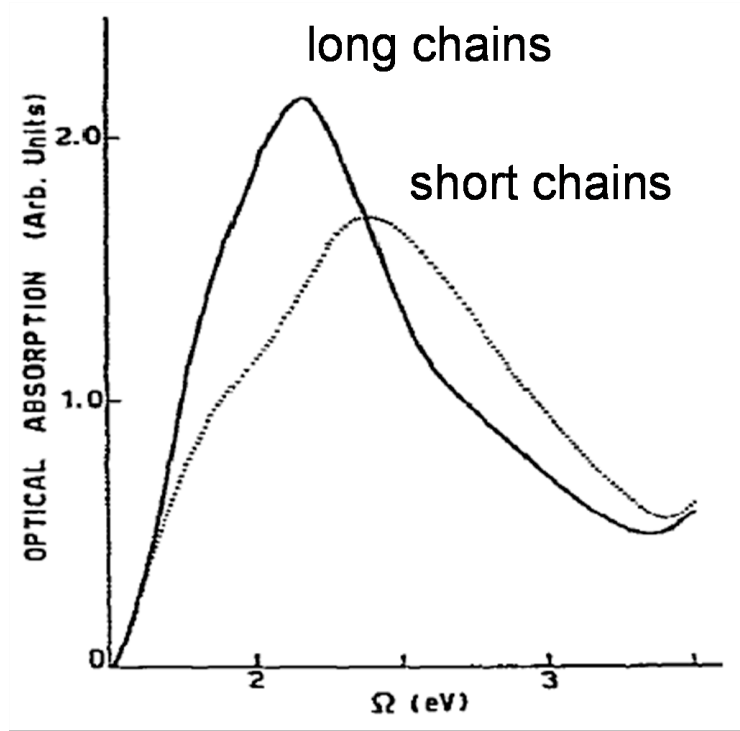

Fig. 6 - Calculated absorption spectra from long and short chains constituting trans$(\mathrm{CH})_{\mathrm{x}}$ samples, adopted from Ref. 16.

$I\left(\omega_{3}\right)$ shown in Fig. 4 and the $I\left(\omega_{3}\right) / I\left(\omega_{1}\right)$ ratio approaching 1.0 following the AM calculations graphically represented in Figure 7. This observation suggest that green Raman laser could become a wavelength of choice for natural identification of trans$(\mathrm{CH})_{\mathrm{x}}$ inclusions in carbonaceous materials fitting both $\omega_{1}$ and $\omega_{3}$ contributions at equivalent intensities. 


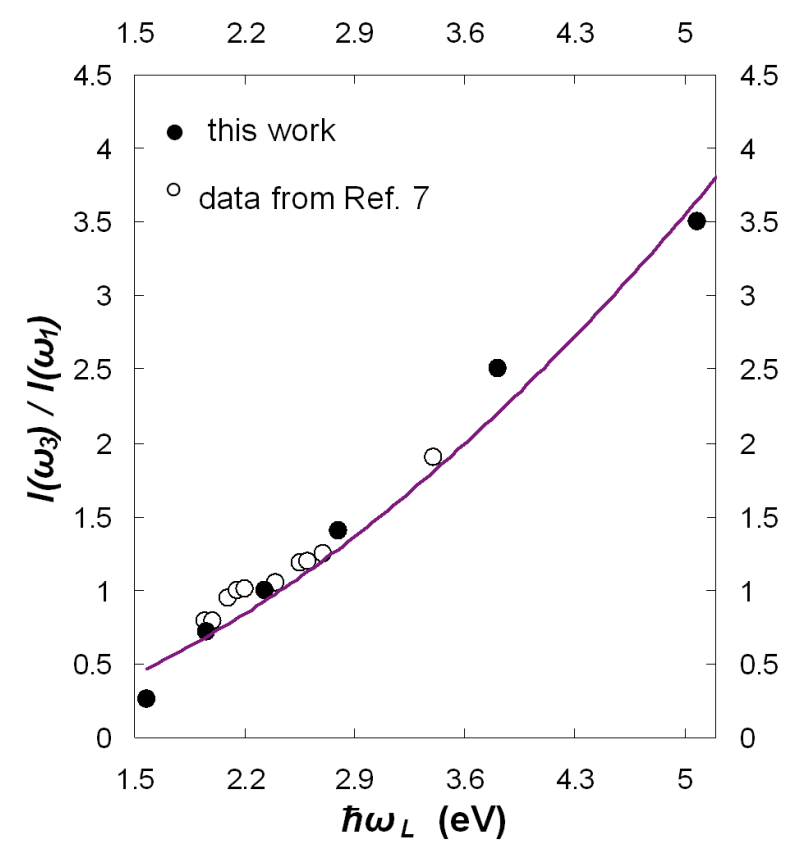

Fig. 7 - The intensity ratio of $I\left(\omega_{3}\right) / I\left(\omega_{1}\right)$ vs. the laser excitation energy $\hbar \omega_{L}$ for trans$(\mathrm{CH})_{\mathrm{x}}$ inclusions in $a-\mathrm{C}: \mathrm{H}$. Solid line is a theoretical calculation performed using the amplitude mode formalism [7].

\subsection{Identification of sp-hybridized inclusions in a-C:H}

The observation that contributions belonging to $s p$-hybridised species could be deducted from the main XPS $C_{1 \mathrm{~s}}$ core level electron spectra were first reported by Sergushin et al. [36] for X-ray studies of "carbyne" that suggested a much lower binding energy (BE) level for an $s p$-allotrope, relative to BE for elemental carbon contributions. Figure 8 shows the broad core-level XPS $C_{1 \mathrm{~s}}$ spectra of $a$-C:H; in order to deduct the information about the relative abundance of carbon hybridised fractions in the examined materials, the spectra were decomposition onto three main constituent 
peaks corresponding to $s p, s p^{2}$ and $s p^{3}$ hybridised states. An $s p$ peak was fitted at an assigned $\mathrm{BE}$ of $283.5 \mathrm{eV}$ in confirmation with previous reports $[26,35,36]$, while $s p^{2}$ and $s p^{3}$ constituents were fitted restricted to their respective energy separation gap, $\Delta \mathrm{BE}, \mathrm{eV}$, defined as the difference between the binding energies of $s p^{3}$ and $s p^{2}$ constituents: $\Delta \mathrm{BE}=\mathrm{BE}_{s p 3}-\mathrm{BE}_{s p 2}$ and with $0.85 \leq \Delta \mathrm{BE} \leq 0.9 \mathrm{eV}[37,55]$ the following binding energy positions were obtained for $s p^{2}$ at $\sim 284.4 \mathrm{eV}$ and $s p^{3}$ at $\sim 285.3 \mathrm{eV}$. Due to ex situ XPS measurements and the exposure of samples to air two secondary peaks were added into the fitting of the main $C_{1 \mathrm{~s}}$ spectra: a single $\mathrm{C}-\mathrm{O}$ peak at $\sim 286.8 \mathrm{eV}$ and a carbonyl $\mathrm{C}=\mathrm{O}$ peak at $\sim 288.5 \mathrm{eV}$. The $s p / s p^{2} / s p^{3}$ value ratio

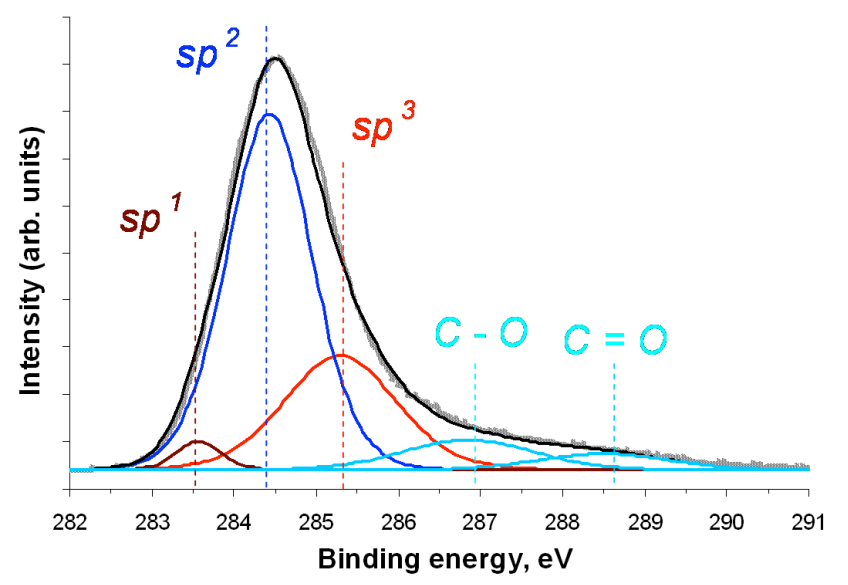

Fig. 8 - XPS core-level $C_{1 \mathrm{~s}}$ spectra of $a-\mathrm{C}: \mathrm{H}$; contributions for $s p, s p^{2}$ and $s p^{3}$ fractions are shown together with $\mathrm{C}-\mathrm{O}$ and $\mathrm{C}=\mathrm{O}$ secondary peaks.

was calculated on the basis of integrating peak areas for the three respective linefunctions and was found to be $0.03 / 0.67 / 0.30$ or, when expressed as a percentage: $3 \%$ $s p, 67 \% s p^{2}$ and $30 \% s p^{3}$; the absolute uncertainty of the measurements was high at 
$\leq 1.25 \%$ owning to variable $\triangle \mathrm{BE}$ gap parameter used in the non-linear least squares fitting procedure. The FWHM for $s p, s p^{2}$ and $s p^{3}$ were found to be correspondingly $\sim 0.6 \mathrm{eV}, \sim 1.2 \mathrm{eV}$ and $\sim 1.6 \mathrm{eV}$, to some extent wider that FWHM values reported for general $a-\mathrm{C}: \mathrm{H}$ or $t a-\mathrm{C}: \mathrm{H}$ materials.

The Raman spectra in the range of $1900-2200 \mathrm{~cm}^{-1}$ are commonly identified with $s p$-hybridised species $[24,25,38,39]$. Figure 9 shows the RRS of examined $a$ $\mathrm{C}: \mathrm{H}$ films; the spectra from $532 \mathrm{~nm}$ to $325 \mathrm{~nm}$ displays only minor perturbations in this range and the $s p$ contributions become clearly visible when probed by $244 \mathrm{~nm}$ laser. Stability of polyyne and cumulene ( $s p$-bonded) species is greatly influenced by

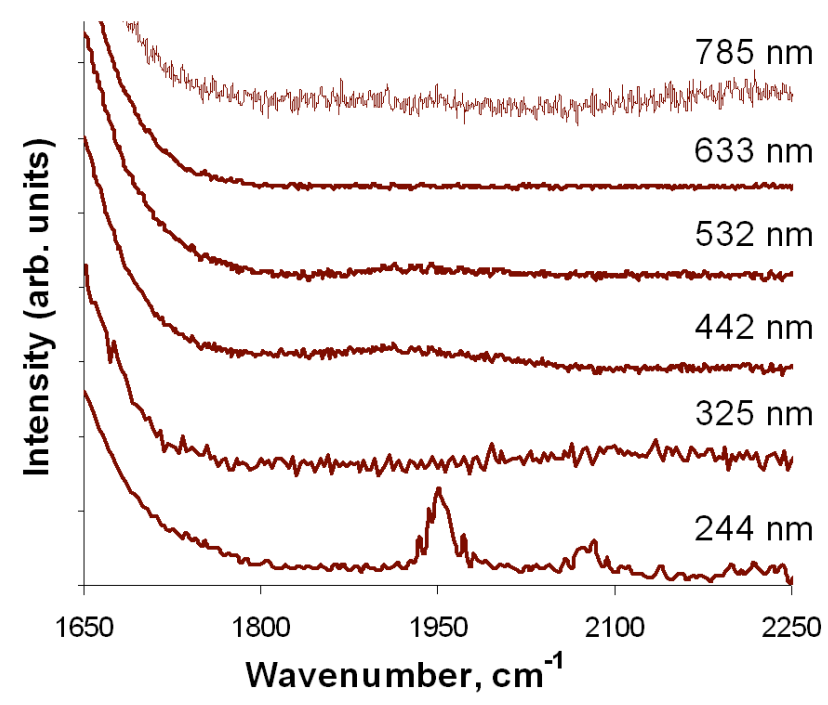

Fig. 9 - Resonant Raman spectra of $s p$-hybridised (polyyne) segments in $a$-C:H.

the hydrogen environment, and polyynes are known to be significantly more resistant to hydrogen exposure [21]. For that reason we consider polyynes to be prevailing 
species in the studied $a$-C:H. Polyynes have been theoretically predicted $[23,56]$ to be more energetically stable than cumulenes, and such preferential stability have been evidenced experimentally $[22,57]$.

Recent works by Tabata et al. $[58,59]$ substantiated the assignment of Raman frequency modes centred at around $2000 \mathrm{~cm}^{-1}$ to hydrogen capped short $H-(C C)_{n}-H$ polyynic chains with $n=8-18$, as did other workers on this subject $[60,61]$. The assignment of the peaks at $\sim 1950 \mathrm{~cm}^{-1}$ and $2070 \mathrm{~cm}^{-1}$ which appeared in the $244 \mathrm{~nm}$ spectrum to fixed-length polyynes is unjustified in our case, since the great majority of published work on the subject considers $s p$ inclusions as completely detached. Satisfactory explanation for observation of polyynes exclusively under UV excitation (Fig. 8) could no longer be regarded exclusively resulting from the resonance enhancement of apparently smaller cross section area of one dimensional $s p$-bonded atoms, as we considered previously [38] relying on D'Urso et al. [19] findings, which suggested nearly an exponential increase of a combined $s p-s p^{2}$ probing signal with respect to increasing $\hbar \omega_{L}$. Recently released $a b$ initio calculations within density functional theory by Ravagnan et al. [62] showed that experimental Raman spectra in the range of $1900-2200 \mathrm{~cm}^{-1}$ for torsionally strained $s p$-nanowires stabilised by $s p^{2}$ and $s p^{3}$ terminations were highly sensitive to strain; changes to the relative orientation of the terminations were found to affect the strain, which subsequently, modulates the electronic states of the nanowires and Raman signal. The appearance of $s p$-bonded species under UV excitation only (VDOS probing) could be attributed to aligned $s p$ hybridised atoms bridging a nanometric gap on $s p^{2}-s p^{3}$ matrix; such $s p$ chains are most likely to be end-terminated by an $s p^{2}$ or an $s p^{3}$ hybridised fragment; $s p$ stabilisation could be achieved in a form of end-termination or bridging. 
Figure 10 illustrates $C-H$ stretching band spectra in the range of $3400-2700$ $\mathrm{cm}^{-1}$ taken from $a-\mathrm{C}: \mathrm{H}$ samples after a baseline correction.

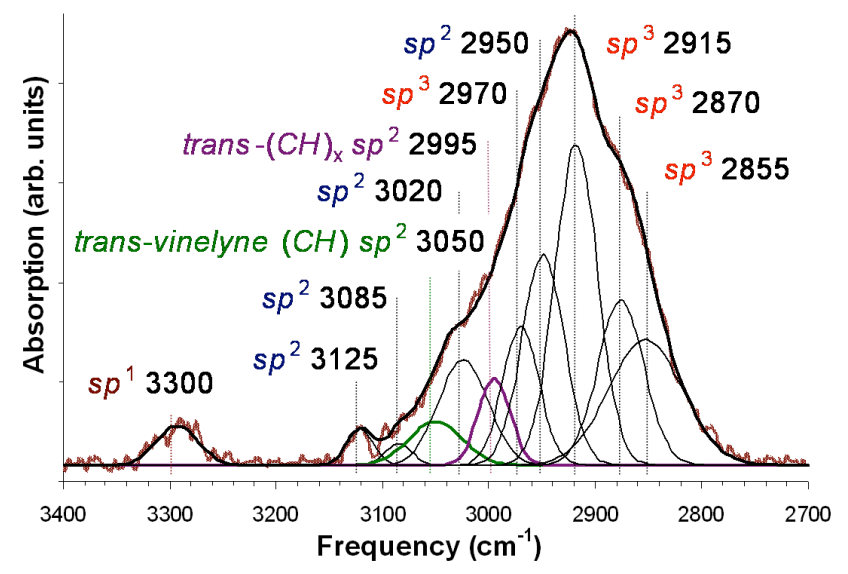

Fig. 10 - Decomposed IR stretching vibrations spectra of an $a-\mathrm{C}: \mathrm{H}$ film. The constituent bonding groups are: $s p^{1}(3300) C H, s p^{2}(3125)=C-H$ unsat/A/asym, $s p^{2}(3085)=C H_{2}$ unsat $/ \mathrm{O} /$ asym, $s p^{2}(3050)=C-H$ sat $/ \mathrm{A} /$ asym, $s p^{2}(3020)$ trans -vinelyne (CH) sat/O/sym, $s p^{2}(2995)$ trans- $(\mathrm{CH})_{\mathrm{x}}$ sat/O/sym, $s p^{3}(2970)-\mathrm{CH}_{3}$ sat/O/asym, $s p^{2}(2950)=\mathrm{CH}_{2}$ sat $/ \mathrm{O} / \mathrm{asym}, s p^{3}(2915)=\mathrm{CH},=\mathrm{CH}_{2}$ sat $/ \mathrm{O} / \mathrm{asym}, \quad s p^{3}(2870)-\mathrm{CH}_{3}$ sat $/ \mathrm{O} / \mathrm{sym}, \mathrm{sp}^{3}(2855)=\mathrm{CH}_{2}$ sat $/ \mathrm{O} / \mathrm{sym}$.

The configuration of constituent groups is shown in abbreviated form as: saturated (sat); unsaturated (unsat); aromatic (A); olefinic (O); symmetric (sym); and asymmetric (asym). The quantitative $[5,6,32,63]$ decomposition reveals contributions from the $s p$-bonded species at $3000 \mathrm{~cm}^{-1}$; the amount of $s p$-hybridised inclusions estimated using Liu et al. [32] calculations never exceeded 3\% for all films deposited. 
The trans- $(\mathrm{CH})_{\mathrm{x}}$ mode is visible at $2995 \mathrm{~cm}^{-1}$, however the infrared absorption of this mode is expected to be significantly lower than other $C-H$ modes fitted, as compared to free molecules. The relative intensity and FWHM of this mode was found closely related to hydrogen content in the films [13]; the mode contribution for the films studied was between 7 to $10 \%$. The stretching mode corresponding to transvinelyne was found at approximately $3020 \mathrm{~cm}^{-1}$ [50]. The amount of $s p$-bonded species in $a-\mathrm{C}: \mathrm{H}$ was found to some extent related to hydrogen content in the films, the ion energy during deposition and deposition temperature; the amount of trans$(\mathrm{CH})_{\mathrm{x}}$ and PPV inclusions was strongly influenced by plasma density and electron temperature.

\section{Summary and conclusions}

In summary, we have performed the RRS investigation on ICP fabricated $a-\mathrm{C}: \mathrm{H}$ films and have demonstrated that the films host trans- $(\mathrm{CH})_{\mathrm{x}}$ segments of various conjugation length, poly( $p$-phenylene vinylene) chains as evidenced by the $1175 \mathrm{~cm}^{-1}$ Raman mode and, a small fraction of $s p$-hybridised carbon species as evidenced under UV excitations. We provided the theoretical basis for arguing that at various laser excitation energies the changes in Raman spectra features for trans- $(\mathrm{CH})_{\mathrm{x}}$ segments included in the $a$-C:H matrix are practically identical to the changes observed in bulk trans-polyacetylene. This leads to reliable identification of trans $-(\mathrm{CH})_{\mathrm{x}}$ inclusions in Raman spectra of $a-\mathrm{C}: \mathrm{H}$ or any other complex carbonaceous material and, differentiation between the Raman active modes for trans $-(\mathrm{CH})_{\mathrm{x}}$ and PPV. 
We observed that relative intensities of trans- $(\mathrm{CH})_{\mathrm{x}}$ core $A_{g}$ modes $\left(\omega_{1}\right.$ and $\left.\omega_{3}\right)$ are essentially equal when probed by excitation energy of green light laser. This is although supported by the theoretical distribution of $I\left(\omega_{3}\right) / I\left(\omega_{1}\right)$ ratio relative to Raman excitation energy, overall exemplifying the approach for trans $-(\mathrm{CH})_{\mathrm{x}}$ identification in carbonaceous solids.

The length of trans- $(\mathrm{CH})_{\mathrm{x}}$ segments in the films examined was found averaging $\sim 25$ ( \pm 5$) C=C$ bond length units, with longer chains of up to 120 bond length units probed by NIR and shorter chains of $\sim 8$ units probed by UV $\hbar \omega_{L}$; all trans- $(\mathrm{CH})_{\mathrm{x}}$ inclusions irrespective of conjugation length displayed high degree of bonding disorder. We assigned the $1175 \mathrm{~cm}^{-1}$ peak to PPV $C C-H$ bending mode of the ring and postulated the origin of this mode. $S p$-hybridised species observed exclusively under UV excitation were identified as short polyynic chains bridging a nanometric gap on $s p^{2}-s p^{3}$ matrix; and these chains were believed to be end-terminated by an $s p^{2}$ or an $s p^{3}$ hybridised fragments.

The presence of $s p$-bonded inclusions in $a$-C:H was confirmed by means of XPS $C_{1 \mathrm{~s}}$ core-electron spectra analysis, while the existence of $s p$, trans $-(\mathrm{CH})_{\mathrm{x}}$ inclusions and trans-vinelyne segments was verified by FT-IR analysis.

Finally, the inclusions of basic polymeric chains such as long trans $-(\mathrm{CH})_{\mathrm{x}}$ and PPV were possible owning to highly ordered $s p^{2}$ host component of $a-\mathrm{C}: \mathrm{H}$ films. Such $s p^{2}$ ordering was achieved via fabrication of $a-\mathrm{C}: \mathrm{H}$ films in ICP reactor with high plasma density and low electron temperature compared to conventional DLC deposition systems. A parallel could be drawn with the report by Chen et al. [64] where unusual inclusions of silicon based spherical nanocrystallites into DLC matrix was possible due to similar ICP fabrication conditions, whereas the stability of $s p$ - 
hybridised species in $a-\mathrm{C}: \mathrm{H}$ could be attributed to relatively low temperature deposition process.

\section{Acknowledgements}

The authors are thankful to G. Hope of Griffith University and S. Prawer of the University of Melbourne for assisting with the UV Raman measurements. M.R. is grateful to L. Ravagnan and P. Milani of the University of Milan and members of the Laboratorio Getti Molecolari for valuable discussions. M.R. acknowledges the BAM for the visiting postdoctoral fellowship funding. This work has been supported by the Australian Research Council and Australian Nano TAP program. 


\section{References}

[1] Bernasconi M, Parrinello M, Chiarotti GL, Focher P, Tosatti E. Anisotropic aC:H from Compression of Polyacetylene. Physical Review Letters. 1996;76(12):2081. [2] Arbuckle GA, MacDiarmid AG, Lefrant S, Verdon T, Mulazzi E, Brivio GP, et al. Optical spectroscopic investigation of segmented trans-polyacetylene. Physical Review B. 1991;43(6):4739.

[3] Robertson J. Diamond-like amorphous carbon. Materials Science and Engineering: R. 2002;37(4-6):129-281.

[4] López-Ríos T, Sandré É, Leclercq S, Sauvain É. Polyacetylene in Diamond Films Evidenced by Surface Enhanced Raman Scattering. Physical Review Letters. 1996;76(26):4935-8.

[5] Dischler B, Bubenzer A, Koidl P. Bonding in hydrogenated hard carbon studied by optical spectroscopy. Solid State Communications. 1983 1983/10;48(2):105-8.

[6] Piazza F, Golanski A, Schulze S, Relihan G. Transpolyacetylene chains in hydrogenated amorphous carbon films free of nanocrystalline diamond. Applied Physics Letters. 2003;82(3):358-60.

[7] Ehrenfreund E, Vardeny Z, Brafman O, Horovitz B. Amplitude and phase modes in trans-polyacetylene: Resonant Raman scattering and induced infrared activity. Physical Review B. 1987;36(3):1535 LP - 53.

[8] Brivio GP, Mulazzi E. Theoretical analysis of absorption and resonant Raman scattering spectra of trans-(CH)x. Physical Review B. 1984;30(2):876.

[9] Ferrari A, Robertson J. Origin of the $1150 \mathrm{~cm}-1$ Raman mode in nanocrystalline diamond. Physical Review B. 2001;63:121405. 
[10] Pfeiffer R, Kuzmany H, Knoll P, Bokova S, Salk N, Gunther B. Evidence for trans-polyacetylene in nano-crystalline diamond films. Diamond and Related Materials, 13th European Conference on Diamond, Diamond-Like Materials, Carbon Nanotubes, Nitrides and Silicon Carbide. 2003;12(3-7):268-71.

[11] Kuzmany H, Pfeiffer R, Salk N, Gunther B. The mystery of the $1140 \mathrm{~cm}-1$ Raman line in nanocrystalline diamond films. Carbon, European Materials Research Society 2003, Symposium B: Advanced Multifunctional Nanocarbon Materials and Nanosystems. 2004;42(5-6):911-7.

[12] Michaelson S, Ternyak O, Hoffman A, Lifshitz Y. Hydrogen incorporation processes in nanodiamond films studied by isotopic induced modifications of Raman spectra. Applied Physics Letters. 2006;89(13):131918.

[13] Teii K, Ikeda T, Fukutomi A, Uchino K. Effect of hydrogen plasma exposure on the amount of trans-polyacetylene in nanocrystalline diamond films. Journal of Vacuum Science and Technology B. 2006;24(1):263-6.

[14] Rybachuk M, Hu A, Bell JM. Resonant Raman scattering from polyacetylene and poly(p-phenylene vinylene) chains included into hydrogenated amorphous carbon. Applied Physics Letters. 2008;93(5):051904-3.

[15] Mullazzi E, Brivio GP, Faulques E, Lefrant S. Experimental and theoretical Raman results in trans polyacetylene. Solid State Communications. 1983;46(12):8515.

[16] Brivio GP, Mulazzi E. Absorption and resonant Raman scattering from trans(CH)x. Chemical Physics Letters. 1983;95(6):555-60.

[17] Ferrari AC, Robertson J. Resonant Raman spectroscopy of disordered, amorphous, and diamondlike carbon. Physical Review B. 2001;64(7):075414. 
[18] Heeger AJ, Kivelson S, Schrieffer JR, Su W-P. Solitons in conducting polymers. Reviews of Modern Physics. 1988;60(3):781-850.

[19] D'Urso L, Compagnini G, Puglisi O. $\mathrm{sp} / \mathrm{sp} 2$ bonding ratio in sp rich amorphous carbon thin films. Carbon. 2006;44(10):2093-6.

[20] Heimann RB, Kleiman J, Salansky NM. A unified structural approach to linear carbon polytypes. Nature. 1983;306(5939):164-7.

[21] Lenardi C, Barborini E, Briois V, Lucarelli L, Piseri P, Milani P. NEXAFS characterization of nanostructured carbon thin-films exposed to hydrogen. Diamond and Related Materials. 2001 2001/0;10(3-7):1195-200.

[22] Agostino RG, Caruso T, Chiarello G, Cupolillo A, Pacile D, Filosa R, et al. Thermal annealing and hydrogen exposure effects on cluster-assembled nanostructured carbon films embedded with transition metal nanoparticles. Physical Review B. 2003;68(3):035413-12.

[23] Springborg M, Kavan L. On the stability of polyyne. Chemical Physics. 1992 1992/12/15;168(2-3):249-58.

[24] Ravagnan L, Siviero F, Lenardi C, Piseri P, Barborini E, Milani P, et al. Cluster-Beam Deposition and in situ Characterization of Carbyne-Rich Carbon Films. Physical Review Letters. 2002;89(28):285506-4.

[25] Hu A, Rybachuk M, Lu QB, Duley WW. Direct synthesis of sp -bonded carbon chains on graphite surface by femtosecond laser irradiation. Applied Physics Letters. 2007;91(13):131906.

[26] Hu A, Griesing S, Rybachuk M, Lu Q-B, Duley WW. Nanobuckling and x-ray photoelectron spectra of carbyne-rich tetrahedral carbon films deposited by femtosecond laser ablation at cryogenic temperatures. Journal of Applied Physics. 2007;102(7):074311-6. 
[27] Heimann RB. Linear finite carbon chains (carbynes): their role during dynamic transformation of graphite to diamond, and their geometric and electronic structure. Diamond and Related Materials. 1994;3(9):1151-7.

[28] Baughman RH. Dangerously Seeking Linear Carbon. Science. 2006;312(5776):1009-110.

[29] Varga IK. Multipurpose plasma generator suitable for diamondlike carbon film formation. J Vac Sci Technol A. 1989;7(4):2639-45.

[30] Rybachuk M, Bell JM. The observation of $\mathrm{sp} 2$ fraction disorder using dual wavelength Raman spectroscopy in a-C:H films fabricated using an open inductively coupled plasma reactor. Diamond and Related Materials. 2006;15(4-8):977-81.

[31] Stoney GG. The Tension of Metallic Films Deposited by Electrolysis. Proceedings of the Royal Society London A. 1909 May 6;82(82):172 - 5.

[32] Liu S, Gangopadhyay S, Sreenivas G, Ang SS, Naseem HA. Infrared studies of hydrogenated amorphous carbon (a-C:H) and its alloys (a-C:H,N,F). Physical Review B. 1997;55(19):13020.

[33] Casiraghi C, Ferrari AC, Robertson J. Raman spectroscopy of hydrogenated amorphous carbons. Physical Review B. 2005;72(8):085401.

[34] Shirley DA. High-resolution X-ray photoemission spectrum of the valence bands of gold. Physical Review B (Solid State). 1972;5(12):4709-14.

[35] Zhang L, Ma H, Yao N, Lu Z, Zhang B. Growth and field electron emission properties of nanostructured white carbon films. Journal of Vacuum Science \& Technology B: Papers from the 19th International Vacuum NanoElectronics conference. 2007;25:545-7. 
[36] Sergushin IP, Kudryavtsev YP, Élizen VM, Sadovskii AP, Sladkov AM, Nefedov VI, et al. X-ray electron and X-ray spectral study of carbyne. Journal of Structural Chemistry. 1977;18(4):553-5.

[37] Haerle R, Riedo E, Pasquarello A, Baldereschi A. sp[sup 2]/sp[sup 3] hybridization ratio in amorphous carbon from $\mathrm{C}$ 1s core-level shifts: X-ray photoelectron spectroscopy and first-principles calculation. Physical Review B. 2002;65(4):045101.

[38] Hu A, Lu Q-B, Duley WW, Rybachuk M. Spectroscopic characterization of carbon chains in nanostructured tetrahedral carbon films synthesized by femtosecond pulsed laser deposition. The Journal of Chemical Physics. 2007;126(15):154705.

[39] $\mathrm{Hu}$ A, Rybachuk M, Lu Q-B, Duley WW. Femtosecond pulsed laser deposition and optical properties of diamond-like amorphous carbon films embedded with sp-bonded carbon chains. Diamond and Related Materials. 2008;17(7-10):16436.

[40] Prawer S, Nugent KW, Lifshitz Y, Lempert GD, Grossman E, Kulik J, et al. Systematic variation of the Raman spectra of DLC films as a function of sp2:sp3 composition. Diamond and Related Materials. 1996;5(3-5):433-8.

[41] Benner DC, Rinsland CP, Devi VM, Smith MAH, Atkins D. A multispectrum nonlinear least squares fitting technique. Journal of Quantitative Spectroscopy and Radiative Transfer. 1995;53(6):705-21.

[42] Brivio GP, Mulazzi E. Theoretical analysis of absorption and resonant Raman scattering spectra of trans-(CH)_\{x\}. Physical Review B. 1984;30(2):876.

[43] Fitchen DB. Resonance raman results in polyacetylene. Molecular Crystals and Liquid Crystals. 1982;83(1):95 - 108. 
[44] Kuzmany H. Resonance Raman Scattering from Neutral and Doped Polyacetylene. Physica Status Solidi B. 1980;97(2):521-31.

[45] Lanzani G, Luzzati S, Tubino R, Dellepiane G. Polarized resonant Raman scattering of cis polyacetylene. The Journal of Chemical Physics. 1989;91(2):732-7.

[46] Mulazzi E. Polarized resonant Raman scattering spectra from stretched trans polyacetylene. Theory. Solid State Communications. 1985;55(9):807-10.

[47] Baitoul M, Wery J, Buisson J-P, Arbuckle G, Shah H, Lefrant S, et al. In situ resonant Raman and optical investigations of p-doped poly ( $p$-phenylene vinylene). Polymer. 2000;41:6955-64.

[48] Tzolov M, Koch VP, Bruetting W, Schwoerer M. Optical characterization of chemically doped thin films of poly p-phenylene vinylene/. Synthetic Metals. 2000;109:85-9.

[49] Orion I, Buisson J-P, Lefrant S. Spectroscopic studies of polaronic and bipolaronic species in n-doped poly(paraphenylenevinylene). Physical Review B. 1998;57(12):7050.

[50] Bradley DDC. Precursor-route poy(p-phenylenevinilene): polymer characterisation and control of electronic properties. Journal of Physics D: Applied Physics. 1987;20:1389 - 410.

[51] Lichtmann LS, Fitchen DB, Temkin H. Resonant Raman spectroscopy of conducting organic polymers. $(\mathrm{CH}) \mathrm{x}$, and an oriented analog. Synthetic Metals. 1980;1(2):139-49.

[52] Kuzmany H. The particle in the box model for resonance Raman scattering in polyacetylene. Pure and Applied Chemistry. 1985;57(2):235 - 46. 
[53] Tiziani R, Brivio GP, Mulazzi E. Resonant Raman scattering spectra of trans(CD)_ $\{\mathrm{x}\}$ : Evidence for a distribution of conjugation lengths. Physical Review B. $1985 ; 31(6): 4015$.

[54] Vardeny Z, Ehrenfreund E, Brafman O, Horovitz B. Resonant Raman Scattering from Amplitude Modes in trans-(CH)x and $-(\mathrm{CD}) \mathrm{x}$. Physical Review Letters. 1983;51(25):2326.

[55] Díaz J, Paolicelli G, Ferrer S, Comin F. Separation of the sp3 and sp2 components in the C1s photoemission spectra of amorphous carbon films. Physical Review B. 1996;54(11 -15):8064-9.

[56] Kertesz M, Koller J, Azman A. Ab initio Hartree--Fock crystal orbital studies. II. Energy bands of an infinite carbon chain. The Journal of Chemical Physics. 1978;68(6):2779-82.

[57] Casari CS, Bassi AL, Ravagnan L, Siviero F, Lenardi C, Piseri P, et al. Chemical and thermal stability of carbyne-like structures in cluster-assembled carbon films. Physical Review B. 2004;69(7):075422-7.

[58] Tabata H, Fujii M, Hayashi S. Surface-enhanced Raman scattering from polyyne solutions. Chemical Physics Letters. 2006 2006/3/10;420(1-3):166-70.

[59] Tabata H, Fujii M, Hayashi S, Doi T, Wakabayashi T. Raman and surfaceenhanced Raman scattering of a series of size-separated polyynes. Carbon. 2006 2006/12;44(15):3168-76.

[60] Casari CS, Bassi AL, Baserga A, Ravagnan L, Piseri P, Lenardi C, et al. Lowfrequency modes in the Raman spectrum of sp-sp[sup 2] nanostructured carbon. Physical Review B. 2008;77(19):195444-7.

[61] Ravagnan L, Bongiorno G, Bandiera D, Salis E, Piseri P, Milani P, et al. Quantitative evaluation of $\mathrm{sp} / \mathrm{sp} 2$ hybridization ratio in cluster-assembled carbon films 
by in situ near edge X-ray absorption fine structure spectroscopy. Carbon. 2006;44(8):1518-24.

[62] Ravagnan L, Manini N, Cinquanta E, Onida G, Sangalli D, Motta C, et al. Sp carbon nanowires experiencing axial torsion. arXiv:09022573. 200915 February 2009.

[63] Rybachuk M, Bell JM. The effect of sp2 fraction and bonding disorder on micro-mechanical and electronic properties of a-C:H films. Thin Solid Films. 2007;515(20-21):7855-60.

[64] Chen L-Y, Hong FC-N. Diamond-like carbon nanocomposite films. Applied Physics Letters. 2003;82(20):3526-8. 


\section{Figure captions}

Fig. 1 - Resonant Raman spectra of bulk trans- $(\mathrm{CH})_{\mathrm{x}}$ at $78 \mathrm{~K}$ taken for different laser excitation wavelengths. a) $\omega_{L}=457.9 \mathrm{~nm}$; b) $\omega_{L}=514.5 \mathrm{~nm}$; ) $\omega_{L}=600 \mathrm{~nm}$; d) $\omega_{L}$ $=676.4 \mathrm{~nm}$, adapted from Ref. 15 .

Fig. 2 - Resonant Raman spectra of $a-\mathrm{C}: \mathrm{H}$ at $293 \mathrm{~K}$ showing contributions from trans$(\mathrm{CH})_{\mathrm{x}}\left(\omega_{1}\right.$ and $\left.\omega_{3}\right)$ modes, PPV $\left(1175 \mathrm{~cm}^{-1}\right.$ mode $)$, and DLC ( $D, G$, and $T$ modes $)$. An asymmetric peak visible at NIR-visible (green) $\hbar \omega_{L}$ at $950 \mathrm{~cm}^{-1}$ is the second order Si, from Ref. 14.

Fig. 3 a) - Peak dispersion, $\Delta \omega$ and b) peak widths, $\Gamma$ for all constituent peaks as a function of the laser excitation energy $\hbar \omega_{L}$, from Ref. 14 .

Fig. 4 - Changes in the fitted $\omega_{1}, \omega_{3}$ and $1175 \mathrm{~cm}^{-1}$ bands (intensity magnified by a factor of 5) relative to the laser excitation energy $\hbar \omega_{L}$ in the spectra of $a-\mathrm{C}: \mathrm{H}$. Light dotted line over $\omega_{1}$ and $\omega_{3}$ bands denotes the $I\left(\omega_{3}\right) / I\left(\omega_{1}\right)$ trend.

Fig. 5 a) - Evolution of relative intensities of the $1175 \mathrm{~cm}^{-1}$ peak, $I(1175)$ and trans$(\mathrm{CH})_{\mathrm{x}}$ contributions, $I \Sigma\left(\omega_{1}, \omega_{3}\right)$ and b) relative intensities of $\omega_{1}, I\left(\omega_{1}\right)$ and $\omega_{3}, I\left(\omega_{3}\right)$ peaks as a function of the laser excitation energy $\hbar \omega_{L}$. 
Fig. 6 - Calculated absorption spectra from long and short chains constituting trans$(\mathrm{CH})_{\mathrm{x}}$ samples, adopted from Ref. 16.

Fig. 7 - The intensity ratio of $I\left(\omega_{3}\right) / I\left(\omega_{1}\right)$ vs. the laser excitation energy $\hbar \omega_{L}$ for trans$(\mathrm{CH})_{\mathrm{x}}$ inclusions in $a-\mathrm{C}: \mathrm{H}$. Solid line is a theoretical calculation performed using the amplitude mode formalism [7].

Fig. 8 - XPS core-level $C_{1 \mathrm{~s}}$ spectra of $a-\mathrm{C}: \mathrm{H}$; contributions for $s p, s p^{2}$ and $s p^{3}$ fractions are shown together with $\mathrm{C}-\mathrm{O}$ and $\mathrm{C}=\mathrm{O}$ secondary peaks.

Fig. 9 - Resonant Raman spectra of $s p$-hybridised (polyyne) segments in $a$-C:H.

Fig. 10 - Decomposed IR stretching vibrations spectra of an $a-\mathrm{C}: \mathrm{H}$ film. The constituent bonding groups are: $s p^{1}(3300) C H, s p^{2}(3125)=C-H$ unsat/A/asym, $s p^{2}(3085)=\mathrm{CH}_{2}$ unsat $/ \mathrm{O} /$ asym, $s p^{2}(3050)=C-H$ sat $/ \mathrm{A} /$ asym, $s p^{2}(3020)$ trans -vinelyne (CH) sat/O/sym, $s p^{2}(2995)$ trans- $(\mathrm{CH})_{\mathrm{x}}$ sat/O/sym, $s p^{3}(2970)-\mathrm{CH}_{3}$ sat/O/asym, $s p^{2}(2950)=\mathrm{CH}_{2}$ sat $/ \mathrm{O} / \mathrm{asym}, s p^{3}(2915)=\mathrm{CH},=\mathrm{CH}_{2}$ sat $/ \mathrm{O} / \mathrm{asym}, \mathrm{sp}^{3}(2870)-\mathrm{CH}_{3}$ sat $/ \mathrm{O} / \mathrm{sym}, \mathrm{sp}^{3}(2855)=\mathrm{CH}_{2}$ sat $/ \mathrm{O} / \mathrm{sym}$. 